\title{
Quantitative Classification of Quartz by Laser Induced Breakdown Spectroscopy in Conjunction with Discriminant Function Analysis
}

\author{
A. Ali, ${ }^{1,2}$ M. Z. Khan, ${ }^{1}$ I. Rehan, ${ }^{1}$ K. Rehan, ${ }^{1,3}$ and R. Muhammad \\ ${ }^{1}$ Department of Applied Physics, Federal Urdu University of Arts, Science and Technology, Islamabad 44,000, Pakistan \\ ${ }^{2}$ National Center for Physics, Quaid-i-Azam University Campus, Islamabad 44,000, Pakistan \\ ${ }^{3}$ International College, UCAS, Beijing 100190, China
}

Correspondence should be addressed to I. Rehan; irehanyousafzai@gmail.com

Received 9 July 2016; Accepted 31 October 2016

Academic Editor: Jau-Wern Chiou

Copyright (C) 2016 A. Ali et al. This is an open access article distributed under the Creative Commons Attribution License, which permits unrestricted use, distribution, and reproduction in any medium, provided the original work is properly cited.

\begin{abstract}
A responsive laser induced breakdown spectroscopic system was developed and improved for utilizing it as a sensor for the classification of quartz samples on the basis of trace elements present in the acquired samples. Laser induced breakdown spectroscopy (LIBS) in conjunction with discriminant function analysis (DFA) was applied for the classification of five different types of quartz samples. The quartz plasmas were produced at ambient pressure using Nd:YAG laser at fundamental harmonic mode $(1064 \mathrm{~nm})$. We optimized the detection system by finding the suitable delay time of the laser excitation. This is the first study, where the developed technique (LIBS+DFA) was successfully employed to probe and confirm the elemental composition of quartz samples.
\end{abstract}

\section{Introduction}

Quartz is one of the most abundant minerals found on the earth after feldspar. The crystal structure of quartz is a continuous arrangement of $\mathrm{SiO} 4$ silicon-oxygen tetrahedra with every oxygen particle being shared between two tetrahedra, thereby giving a general chemical formula of $\mathrm{SiO} 2$. Quartz has many types according to impurities present in it. Basically, it occurs in all environments and is an essential constituent of many rocks. Previously quartz was classified on the basis of its physical appearance and the possibility of determination of its structural essentials using an optical microscope. A literature survey showed that different experiments have been performed to study the quartz crystals. Laser induced breakdown spectroscopy was used as a rapid tool for the discrimination and analysis of minerals and geological samples [1]. For the quantitative analysis of quartz minerals, a digital analytical system was developed and applied [2]. Laser spectroscopy in conjunction with the Raman spectroscopy was also used for identifying the composition of different minerals. Due to its high power of laser energy, LIBS was also applied for the investigation and interpretation of lithium in pegmatite minerals [3]. This technique was also utilized for the identification of polymer based samples $[4,5]$. To improve the capability of LIBS technique, it has been employed in combination with discriminant function analysis to identify and differentiate between different kinds of polymers [6]. Similarly, the combination of LIBS and DFA was also applied to analyze the Pseudomonas aeruginosa bacteria's colony in blood [7]. The calibration-free LIBS (CF-LIBS) method can be considered an important tool but it still requires more experimental efforts for getting recognized as a useful tool for practical applications [8].

Laser induced breakdown spectroscopy (LIBS) is a multipurpose method fit for analyzing the sample in its original structure and thereby catching up from their point of limitations of other methods in terms of long sample preparation that could lead to the amendment of the chemical composition of the original test sample. The emission spectrum is a finger-print of any material and it can provide a complete elemental composition of any substance [9].

We applied laser induced breakdown spectroscopy in coincidence with the discriminant function analysis for the quantitative investigation of five different types of quartz 


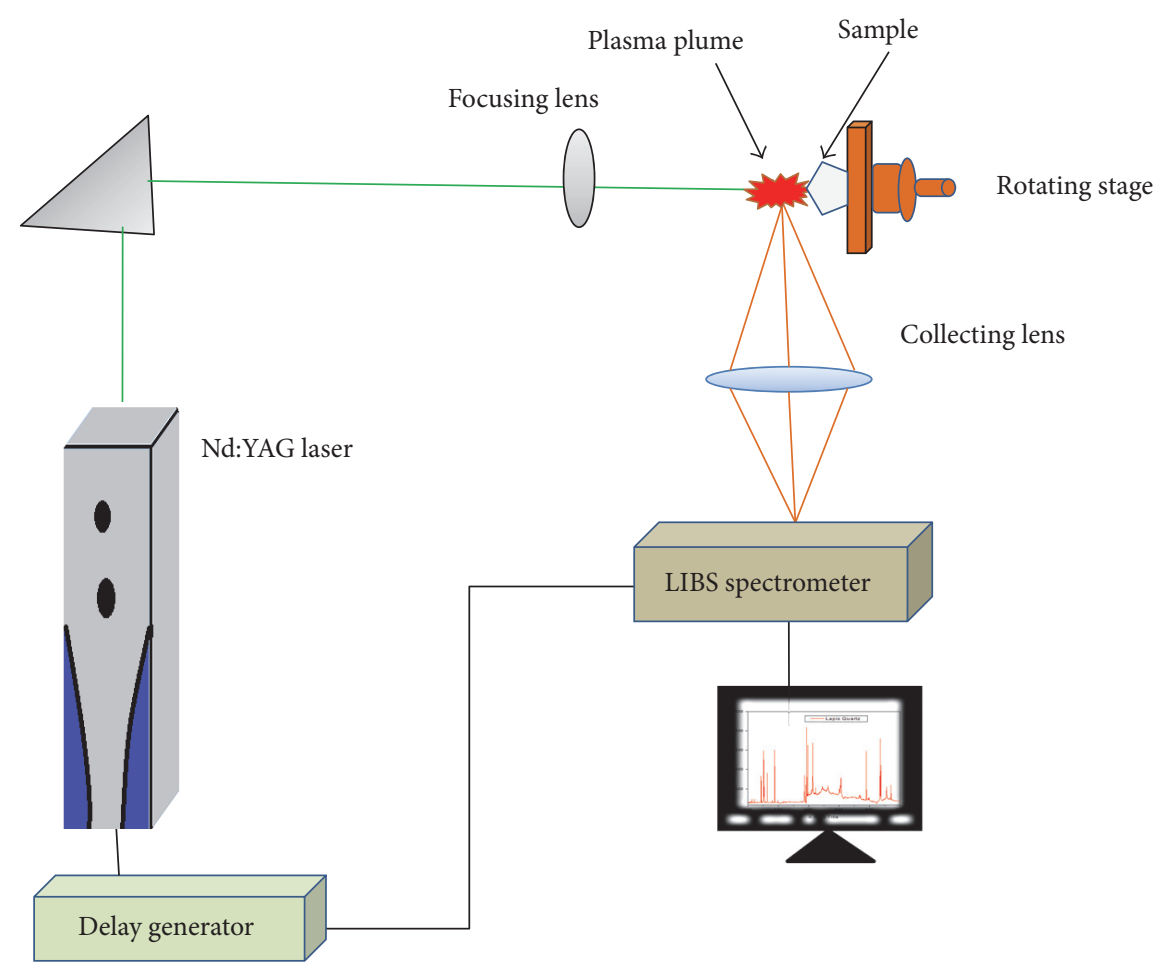

FIGURE 1: Schematic diagram of LIBS setup.

samples, namely, crystal quartz, lapis quartz, pink quartz, tourmaline quartz, and amethyst quartz. For this purpose the samples were collected from Waziristan (North, South) (crystal quartz), Mohmand Agency (pink quartz), and Gilgit-Baltistan (lapis and tourmaline) areas of Khyber Pakhtunkhwa, Pakistan, and one sample (Amethyst) was collected from Minas Gerais, Brazil, South America (SA). The mountains are rich with precious minerals in Pakistan but lack facilities and scientific methods of exploration of these treasures are not proper. Apparently, all types of quartz are quite similar. They can be classified on the basis of their colors, but the difference in colors can be due to different impurities present in quartz as well as different weather conditions in that area.

In this work, plasmas from quartz were produced utilizing Q-switched Nd:YAG laser at fundamental harmonic mode $(1064 \mathrm{~nm})$ in air. The emission spectra of quartz samples were registered by LIBS setup and major constituents were identified with the help of NIST database [10]. Due to similar elemental composition ( $\mathrm{Na}, \mathrm{Li}, \mathrm{Mn}, \mathrm{Si}$, etc.) of the acquired quartz samples, all the samples were similar qualitatively. Discriminant function analysis (DFA) was employed for calculating the percentage profiles of the observed species in the collected quartz samples which is a strong mathematical tool, and has never been used for the classification of quartz. The developed technique (LIBS+DFA) for the classification of quartz has potential applications in the field of core mining.

\section{Experimental Setup}

The experimental setup used in the present work was illustrated in Figure 1. Briefly, it consists of a Nd:YAG laser
(Brilliant, Quantel, France), having a pulse width of $5 \mathrm{~ns}$ and repetition rate of $10 \mathrm{~Hz}$. The laser beam was focused through a $10 \mathrm{~cm}$ quartz lens on the surface of quartz target. The sample was mounted on a rotating stage, which was rotated continuously using a step motor to provide a fresh target surface after each laser pulse. The emitted radiation from the plume was collected by an optical fiber (high$\mathrm{OH}$, core diameter of $600 \mu \mathrm{m})$ having collimating lens $(0-$ $45^{\circ}$ ) field view placed at right angle to the direction of the plume. The optical fiber was connected to the spectrometer (LIBS2000+, Ocean Optics, USA) of focal length $0.4 \mathrm{~m}$ and spectral resolution $\lambda / \Delta \lambda \cong 5000$. The LIBS detection system was equipped with five HR2000 high-resolution miniature spectrometers each having the slit width of $5 \mu \mathrm{m}$ with 2400 lines/mm gratings thereby covering a spectral range of 200$720 \mathrm{~nm}$. Every spectrometer has 2048-element linear silicon charge coupled device (CCD) array with optical resolution $\approx 0.05 \mathrm{~nm}$. The system was calibrated by recording the wellknown lines of neon, argon, and mercury with $\approx 0.02 \mathrm{~nm}$ measurement uncertainty. All the five spectrometers installed in the LIBS2000 were manufacturer calibrated in efficiency using the DH-2000-CAL standard light source. The pulse energy was varied through OOILIBS software. The laser pulse energy was measured using a dedicated energy meter (NOVA-QTL, P/N 1Z01507, Sr. number 56461). The time delay between the laser firing and the opening of the detection system was optimized and set $\sim 3 \mu \mathrm{m}$.

In order to get well resolved emission spectrum at the early stage of plasma generation, the data acquisition time was optimized and found to be $850 \mathrm{~ns}$. Also the optimal laser energy of $100 \mathrm{~mJ} /$ pulse was chosen to avoid the absorption of laser photon by the plasma plume at the higher end of the 


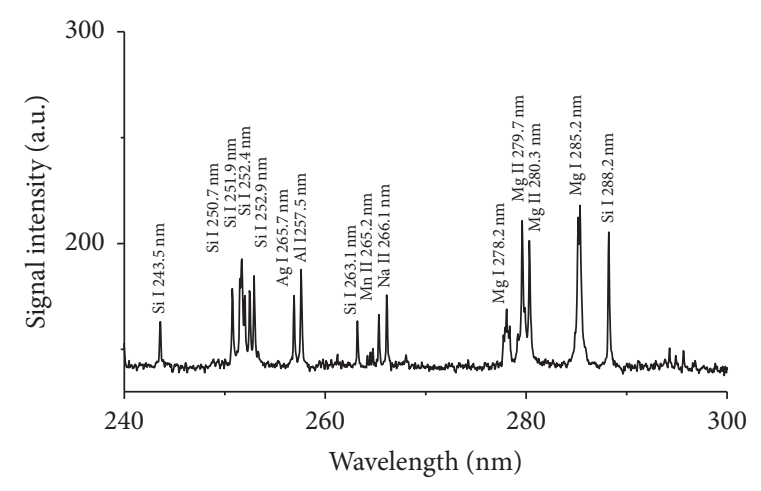

(a)

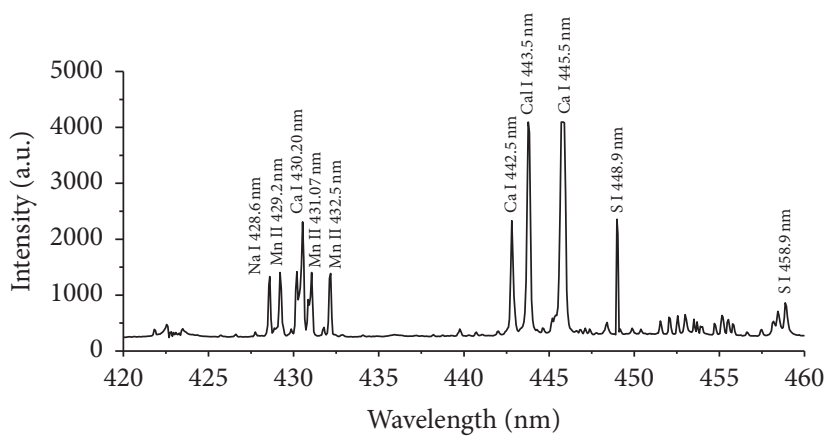

(c)

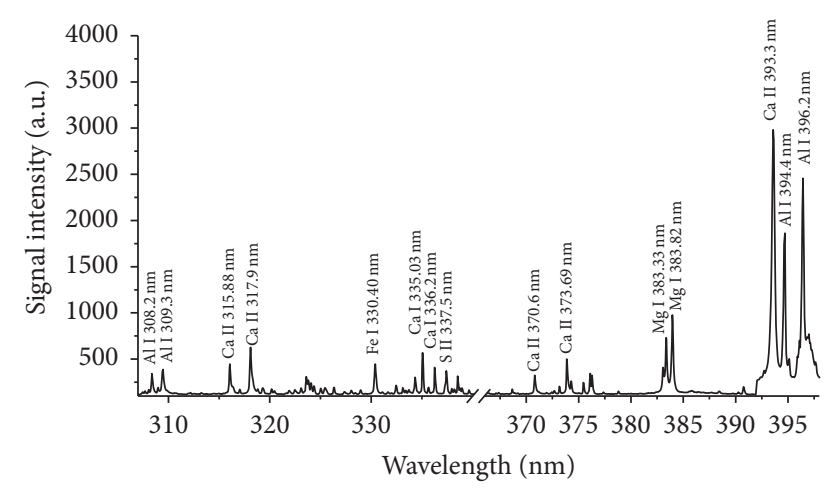

(b)

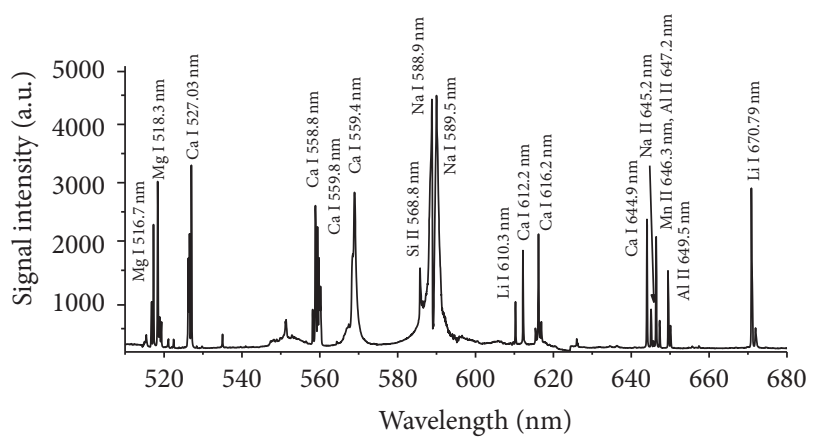

(d)

FIGURE 2: The representative emission spectrum of lapis quartz along with the relevant atomic emission identified, covering a spectral range of $240-680 \mathrm{~nm}$.

laser energy with no compromising on the signal to noise ratio. We used an exposure time of $0.2 \mathrm{~s}$ and a slit width of $50 \mu \mathrm{m}$. To minimize the statistical error, the output data were averaged for 20 laser shots. From the spot diameter of $\sim 103 \mu \mathrm{m}$, a laser fluence of about $44 \mathrm{~J} \mathrm{~cm}^{-2}$ was approximated.

\section{Result and Discussion}

3.1. Study of Emission Spectra. A representative emission spectrum of lapis quartz was shown in Figure 2. For time and space saving we did not show all the emission spectra. To analyze each of the emission spectra we divided each of them into four parts thereby covering a spectral region of $240-680 \mathrm{~nm}$.

The dominant spectral emission lines were atomic and singly ionized lines mainly coming from $\mathrm{Al}, \mathrm{Ca}, \mathrm{Li}$, and $\mathrm{Si}$ and some lines of Fe have been labeled on the graph. Only those strong spectral emissions were taken in calculations which were common in all the emission spectra. The major transitions of different element observed in the emission spectra of quartz were Si I [(243.51), (250.69), (252.41), (252.85), (263.12), (288.15), (298.76)] nm, Li I (670.79 nm), $\mathrm{Na}$ I [(588.995), (589.594), (285.28)] nm, Al I [(394.70), (396.42)] nm, Ca I [(393.62), (397.13), (422.67)] nm, and so forth. The elemental impurities present within quartz such as copper, lithium, silver, and calcium were reported in the literature [11]. The optical emission spectrums of all five different types of quartz were registered and presented in
Figure 3. Due to these similarities, the classification of the collected quartz samples was quite difficult by qualitative analysis of LIBS spectrum. The longest wavelength detectable range of our experimental setup was $720 \mathrm{~nm}$; that is why we were unable to detect oxygen in the collected quartz samples.

A detailed qualitative analysis of the emission spectra of all types of quartz was performed and a small wavelength region of the emission spectrums was presented in Figure 4.

The emission spectrum reveals that, Ca I, II transitions are common in all spectrums, but $\mathrm{Al} \mathrm{I}$ is not present in the emission spectrums of amethyst and crystal quartz. Therefore, on the basis of detailed spectroscopic analysis of emission spectrum, the collected quartz can be classified into two classes of class I (amethyst, crystal quartz) and class II (lapis, pink, and tourmaline). From the relative intensities of $\mathrm{Ca} \mathrm{I}$ and $\mathrm{Al} \mathrm{I}$ emission lines, it can be inferred that the $\mathrm{Ca}$ and $\mathrm{Al}$ concentration could be different in all five types of quartz. However, the complete classification of the quartz samples requires a model for quantitative analysis.

3.2. Discriminant Function Analysis. In last few years, the statistical methods are effectively used in a joint venture with the experiments, especially in the field of laser spectroscopy. Discriminant function analysis (DFA) is one of those statistical techniques which are used to quantify and predict the group membership of more than one group. In this work, we used this method to classify all the established set of observations of the different types of quartz on the basis 


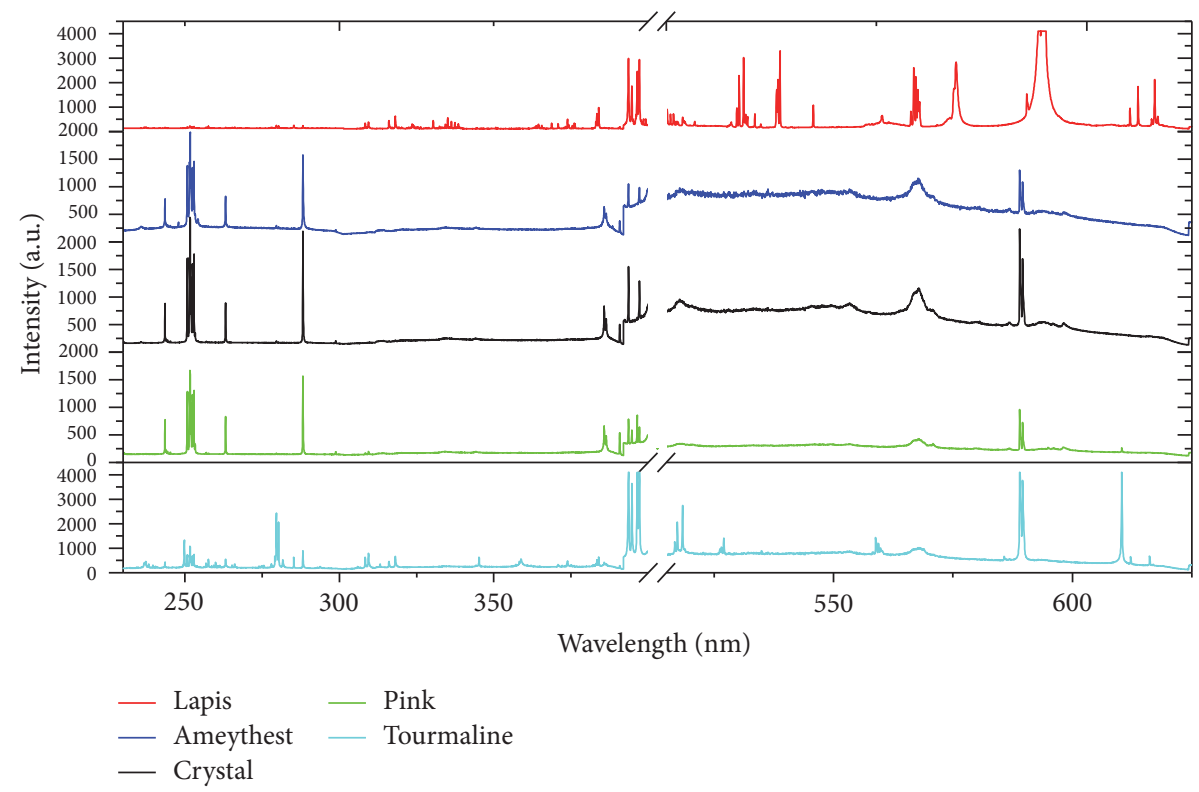

FIGURE 3: Plot showing a comparison between optical emission spectra of all the collected quartz samples.

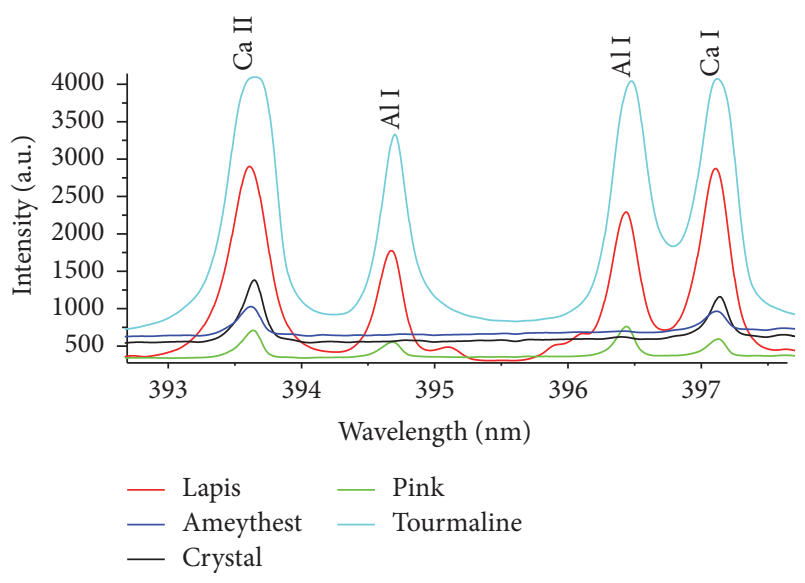

FIgure 4: Optical emission spectrum showing Al I and Ca I, II transition in all five types of quartz.

of independent variable (emission intensities) from each spectrum (individually each spectrum was treated as single input data point) [12].

The mathematical formula for each set of $n$ variables $X_{1}, X_{2}, \ldots, X_{n}$ is given as

$$
D=b_{1} X_{1}+b_{2} X_{2}+\cdots+b_{n} X_{n}+c,
$$

where $D$ is a dependent variable called discriminant function, $X$ 's are the predictive variable or original variable, $b$ 's are the discriminant coefficient or weight for that variable, and $c$ is a constant.

From (1), it is clear that discriminant function is the linear combination of the predictive variables which is responsible for group discrimination. The fundamental motivation behind DFA was to deliver a model to predict the group memberships to figure out the dimensions in which the groups were emphatically differentiated $[6,13]$. In discriminant function, each predictive variable demonstrates its contribution to classify the groups by weighting $b$ 's on that direction. The possible number of discriminant functions (dimension) is less than the number of groups and very nearly equivalent to the predictor variables. DFA point out the established set of $b$ 's to maximize the variance between groups and variance within groups ratios. These functions do not depend upon each other (orthogonal), so it is inferred that discriminations between groups because of these functions do not overlap $[6,12]$. The discriminant function analysis was handled in three stages. In the first stage, canonical correlation analysis generates a set of canonical discriminant functions (shown in Table 2) which are basically the eigenvectors of the observed information and gave a base to maximize the difference between the groups. If there were $N$ groups to be distinguished, then $N-1$ discriminant functions were constructed. The primary functions established a strong differentiation between the groups of all combinations of the variables. The auxiliary function again maximizes the discrimination between groups but at this time secondary function controls for primary factor and so on. In the second stage, a test ( $F$ test) was characterized for the significance in the set of discriminant functions, based on the variances in the groups means, used to ascertain the Wilks' lambda (also called $u$ statistics) whose range is from 0 to 1 . Zero implies that all grouping means are different and one implies that all grouping variables were not distinctive. The last step was the classification, if the result was significant then reject the null hypothesis and the occurrence of classification of the dependent variable appeared by canonical discriminant function analysis of each single data point spectrum [6]. There were five groups of different types of quartz; therefore 
TABLE 1: Variables that are used ${ }^{\mathrm{a}, \mathrm{b}}$ in the analysis at each step.

\begin{tabular}{|c|c|c|c|c|c|c|c|c|c|c|c|c|c|}
\hline \multicolumn{14}{|c|}{ Variables entered/removed ${ }^{\mathrm{a}, \mathrm{b}, \mathrm{c}, \mathrm{d}}$} \\
\hline \multirow{3}{*}{ Step } & \multirow{3}{*}{ Entered } & \multirow{3}{*}{ Statistic } & \multirow{3}{*}{ df1 } & \multirow{3}{*}{ df2 } & \multirow{3}{*}{ df3 } & \multirow{2}{*}{\multicolumn{4}{|c|}{$\begin{array}{l}\text { Wilks' lambda } \\
\text { Exact } F\end{array}$}} & \multirow{2}{*}{\multicolumn{4}{|c|}{ Approximate $F$}} \\
\hline & & & & & & & & & & & & & \\
\hline & & & & & & Statistic & df1 & df2 & Sig. & Statistic & df1 & df2 & Sig. \\
\hline 1 & Si I (243.51)/Li I (670.79) & 0.025 & 1 & 4 & 148.000 & 1419.00 & 4 & 148.000 & 0.000 & & & & \\
\hline 2 & Si I (250.69)/Li I (670.79) & 0.002 & 2 & 4 & 148.000 & 818.586 & 8 & 294.000 & 0.000 & & & & \\
\hline 3 & Si I (243.52)/Na I (588.99 + 589.59) & 0.000 & 3 & 4 & 148.000 & & & & & 590.966 & 12 & 386.571 & 0.000 \\
\hline 4 & Si I (288.15)/Li I (670.79) & 0.000 & 4 & 4 & 148.000 & & & & & 496.521 & 16 & 443.620 & 0.000 \\
\hline 5 & Si I (250.69) Na I (588.99 + 589.59) & 0.000 & 5 & 4 & 148.000 & & & & & 408.230 & 20 & 478.544 & 0.000 \\
\hline 6 & Si I (263.12)/Li I (670.79) & 0.000 & 6 & 4 & 148.000 & & & & & 341.600 & 24 & 500.077 & 0.000 \\
\hline 7 & Si I (288.15)/Na I (588.99 + 589.59) & 0.000 & 7 & 4 & 148.000 & & & & & 293.669 & 28 & 513.410 & 0.000 \\
\hline 8 & Si I (263.12)/Li I (670.79)] & 0.000 & 8 & 4 & 148.000 & & & & & 256.773 & 32 & 521.577 & 0.000 \\
\hline
\end{tabular}

TABle 2: The first 4 canonical discriminant functions used in the analysis.

\begin{tabular}{lcccc}
\hline Function & Eigenvalue & $\begin{array}{c}\text { \% of } \\
\text { variance }\end{array}$ & $\begin{array}{c}\text { Cumulative } \\
\%\end{array}$ & $\begin{array}{c}\text { Canonical } \\
\text { correlation }\end{array}$ \\
\hline 1 & 72.923 & 56.8 & 56.8 & 0.993 \\
2 & 47.804 & 37.2 & 94.0 & 0.990 \\
3 & 7.641 & 6.0 & 100.0 & 0.940 \\
4 & 0.048 & 0.0 & 100.0 & 0.215 \\
\hline
\end{tabular}

four discriminant function scores as well as eight prediction variables were figured out by DFA procedure as tabulated in Table 1. A stepwise DFA was performed to choose a small number of predicators that were responsible for the classification among all the five types of quartz. To know the behavior of the classification a Wilks' lambda was calculated at each step. Wilks' lambda is the ratio of within group sums of square to the sum of square and proportion of total variance in discriminant function scores. We conclude from Table 1 that eight steps were taken and each step included new variable and all were significant as $p<0.000$.

The total discriminant function numbers acquired by DFA process were less than the total groups as $N-1$, where $N$ is the total numbers of groups which were five; therefore, discriminant capacities numbers were four which was significant $(p<0.000)$. For statistical analysis, eight indicators were utilized. The discriminants with their corresponding power of discrimination were tabulated in Table 2. In the first column the eigenvalue is a characteristic background (root) of every function, representing how well the corresponding function distinguishes the groups in the analysis, in such a way that the greater the eigenvalue is the better the discrimination will be. In the $\%$ variance column, we see that the initial three functions have the highest percentage of variance. As inserted in Table 2, the DF1 were contributing $56.8 \%$ of variance between the groups and $37.2 \%$ were contributed by DF2; just $94 \%$ of the variance is shared by the DF1 and DF2. Accumulation of the initial three variances was $100 \%$. The last column of canonical correlation shows the correlation between the group members (dependent variables) and discriminant scores, which represents that these three functions were highly correlated (it ranges from 0 to 1; a high correlation tells us about a function that discriminates well). Therefore the first function was the most imperative function in explanatory power on the grounds that a high correlated predictor contributes more information in the analysis, the second was the next more important, and so on.

Standardized canonical discriminant function coefficients were presented in Table 3, which were playing very important role in discrimination. The plus and minus sign show the direction of relationships. The unique contributions to the discriminant functions were evaluated as can be observed Table 3 . The strongest predictors in the primary function (dimension) were Si I (252.41)/Li I (670.79), Si I $(252.41) / \mathrm{Na} \mathrm{I}(588.99+589.59)$, and $\mathrm{Si} \mathrm{I}(252.85) / \mathrm{Li} \mathrm{I}$ $(670.79) \mathrm{nm}$ scores. The other strongest predictors in secondary function were Si I $(288.15) / \mathrm{Na}$ I $(588.986+589.58)$, Si I (288.15)/Li I (670.79), and Si I (243.51)/Na I (588.986+ $589.58)$ and in the third function column, the next strongest predictors were Si I (252.85)/Li I (670.891), Si I (288.15)/Li I (670.79), Si I (252.85)/Li I (670.79), and so on. The ratios were taken because $\mathrm{Li}$ (at $670.79 \mathrm{~nm}$ ) and $\mathrm{Na}$ (at $588.9 \mathrm{~nm}$, $589.5 \mathrm{~nm}$ ) have a major contribution in the collected samples. The Si I (263.12)/Li I (670.79) and others were the weakest predictors as in Table 3. Hence the powerful factor or strongest predictor which plays an important role in the classification of quartz was [Si I (243.51)/Li I (670.79)] ratio. The classification results were shown in Table 4 , which were based on the DFA. In Table 4, the actual group membership against the predicted group membership was presented. By performing DFA, the quartz was classified in $\sim 99.3 \%$ of the original group cases successfully. To find out the expected accuracy for group membership prediction, we used a leaveone-out method which is also known as jackknife method $[14,15]$. In this method, the identification of each data point was calculated. The process was repeated for all of the observations, leaving out one at each period, and the fact that all of the observations have misclassified case ratios informs us about the actual prediction rate error. In the last, the predicted accuracy was calculated. Therefore using leaveone-out method, all of the correct classifications were done 
TABLE 3: Standardized canonical discriminant function coefficients.

\begin{tabular}{|c|c|c|c|c|}
\hline & \multicolumn{4}{|c|}{ Function } \\
\hline & 1 & 2 & 3 & 4 \\
\hline [Si I (243.51)/Li I (670.79)] & -2.391 & 1.704 & -0.864 & -3.105 \\
\hline [Si I (252.41)/Li I (670.79)] & 7.641 & 0.661 & 0.531 & 3.279 \\
\hline [Si I (252.85)/Li I (670.79)] & -4.620 & -4.877 & 2.149 & -4.737 \\
\hline [Si I (263.12)/Li I (670.79)] & 0.609 & 0.161 & -0.497 & 0.483 \\
\hline [Si I (288.15)/Li I (670.79)] & -0.768 & 3.392 & -1.300 & 4.151 \\
\hline [Si I $(243.51) / \mathrm{Na}$ I $(588.99+589.59)]$ & -3.079 & 2.997 & 6.506 & 0.615 \\
\hline [Si I $(252.41) / \mathrm{Na}$ I $(588.99+589.59)]$ & 5.787 & -8.814 & -2.952 & -2.072 \\
\hline [Si I (288.15)/Na I $(588.99+589.59)]$ & -2.749 & 5.610 & -2.784 & 1.913 \\
\hline
\end{tabular}

TABLE 4: Predicted group membership of quartz and its classification.

\begin{tabular}{|c|c|c|c|c|c|c|c|c|}
\hline \multicolumn{9}{|c|}{ Predicted group membership of quartz } \\
\hline & & ID & Lapis & Crystal & Pink & Tourmaline & Amethyst & Total \\
\hline \multirow{10}{*}{ Original } & \multirow{5}{*}{ Count } & Lapis & 29 & 0 & 0 & 1 & 0 & 30 \\
\hline & & Crystal & 0 & 30 & 0 & 0 & 0 & 30 \\
\hline & & Pink & 0 & 0 & 30 & 0 & 0 & 30 \\
\hline & & Tourmaline & 0 & 0 & 0 & 30 & 0 & 30 \\
\hline & & Amethyst & 0 & 0 & 0 & 0 & 30 & 30 \\
\hline & \multirow{5}{*}{$\%$} & Lapis & 96.8 & 0.0 & 0.0 & 3.2 & 0.0 & 100.0 \\
\hline & & Crystal & 0.0 & 100.0 & 0.0 & 0.0 & 0.0 & 100.0 \\
\hline & & Pink & 0.0 & 0.0 & 100.0 & 0.0 & 0.0 & 100.0 \\
\hline & & Tourmaline & 0.0 & 0.0 & 0.0 & 100.0 & 0.0 & 100.0 \\
\hline & & Amethyst & 0.0 & 0.0 & 0.0 & 0.0 & 100.0 & \\
\hline \multirow{9}{*}{ Cross-validated $^{\mathrm{a}}$} & \multirow{5}{*}{ Count } & Lapis & 29 & 0 & 0 & 1 & 0 & 30 \\
\hline & & Crystal & 0 & 30 & 0 & 0 & 0 & 30 \\
\hline & & Pink & 0 & 0 & 30 & 0 & 0 & 30 \\
\hline & & Tourmaline & 0 & 0 & 0 & 30 & 0 & 30 \\
\hline & & Amethyst & 0 & 0 & 0 & 0 & 30 & 30 \\
\hline & \multirow{4}{*}{$\%$} & Lapis & 96.8 & 0.0 & 0.0 & 3.2 & 0.0 & 100.0 \\
\hline & & Crystal & 0.0 & 100.0 & 0.0 & 0.0 & 0.0 & 100.0 \\
\hline & & Pink & 0.0 & 0.0 & 100.0 & 0.0 & 0.0 & 100.0 \\
\hline & & Tourmaline & 0.0 & 0.0 & 0.0 & 100.0 & 0.0 & 100.0 \\
\hline
\end{tabular}

${ }^{a}$ Cross-validation is done only for those cases in the analysis. In cross-validation, each case is classified by the functions derived from all cases other than that case.

${ }^{\mathrm{b}} 99.3 \%$ of original grouped cases correctly classified.

${ }^{c} 99.3 \%$ of cross-validated grouped cases correctly classified.

99.3\%, which clearly indicates that the conjunction of DFA with LIBS provides a powerful tool for classification of quartz.

The discriminant function scores were calculated by multiplication of the discriminant coefficients and their corresponding predicted variables as given in (1). In Figure 5, the scores of discriminant function 1 and discriminant function 2 were plotted. The same group member's data were scattered about the mean and differences between groups were large as compared to the variance of within groups which are the measure of that scatter (see Figure 5). The amethyst quartz and crystal quartz are somewhat congested relatively at the two neared focuses as shown and characterized from the other three examples. In other words, the complete characterization of every one of the five different types of the collected quartz is impractical by plotting just two function scores. Therefore, a three-dimensional plot of function 1 , function 2, and function 3 scores was exhibited in Figure 6.

Figure 6 demonstrates that the amethyst quartz and crystal quartz were put in the two opposite surfaces of the $3 \mathrm{D}$ plot and the other three are practically at the same line due to the missing of the Al I component. The crystal quartz, pink quartz, and amethyst quartz which were having the weak emission lines of Ca I are clearly separated compared to the other two (tourmaline and lapis quartz).

\section{Conclusion}

The LIBS detection system was improved through finding the suitable delay time between the laser excitation and data acquirement system. LIBS technique in combination 


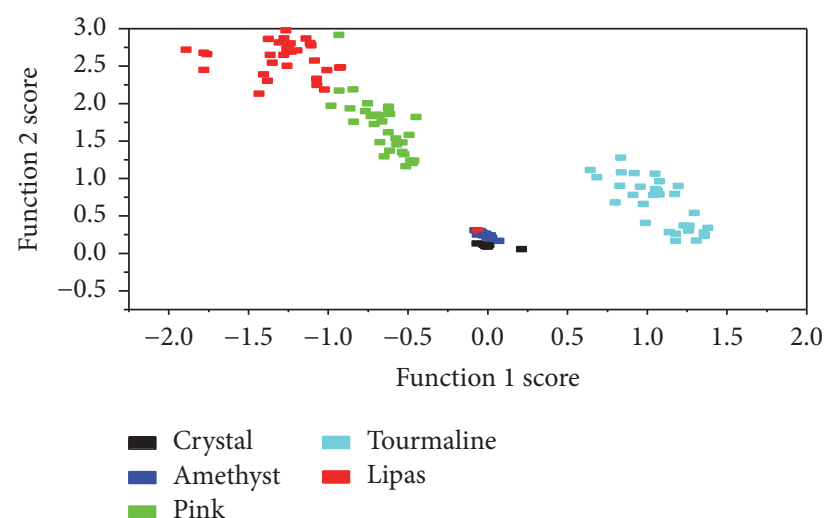

FIgURE 5: Plot showing the comparison between function score 1 and function score 2 .

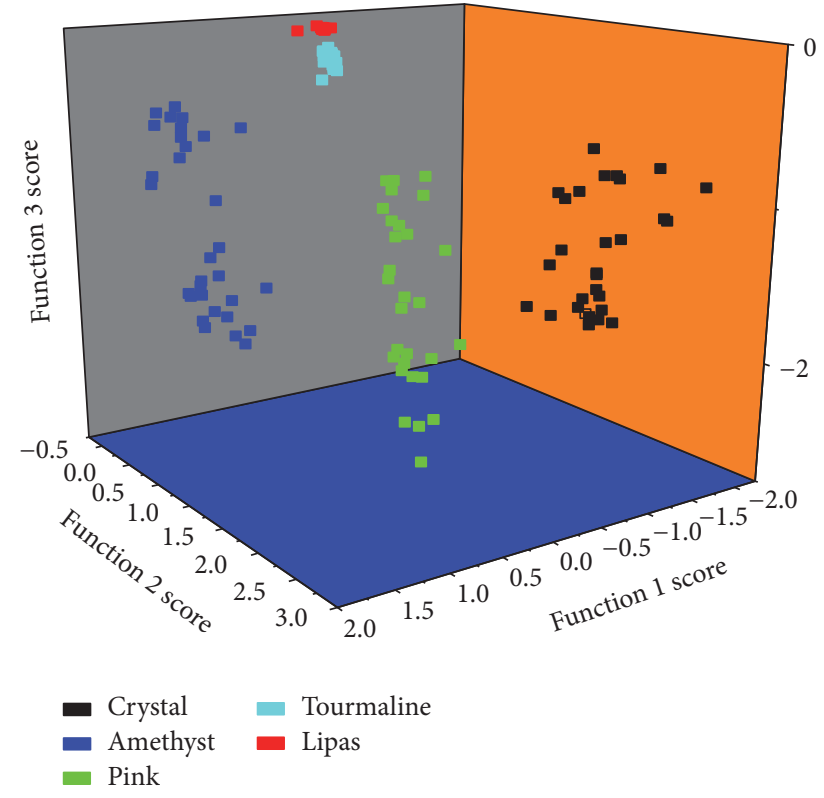

FIGURE 6: 3D plot of all five types of quartz.

with discriminant function analysis (DFA) was employed for the qualitative analysis of five different types of quartz. The strongest predictor in discriminating of quartz was the intensities ratios of Si I (252.41)/Li I (670.79), Si I (252.41)/Na I (588.99+589.59), and Si I (252.85)/Li I (670.79) spectral lines. With the conjunction of DFA and LIBS, the group membership of unknown quartz was predicted $99 \%$ precisely with $1 \%$ predicted error rate. Besides the precise results of LIBS+DFA, it was extremely straightforward, cost effective, and very quick. Therefore, it can be applied for the classification and identification of quartz. The developed technique (LIBS+FDA) was found reliable to investigate the elemental composition of quartz samples.

\section{References}

[1] R. S. Harmon, J. Remus, N. J. McMillan et al., "LIBS analysis of geomaterials: geochemical fingerprinting for the rapid analysis and discrimination of minerals," Applied Geochemistry, vol. 24, no. 6, pp. 1125-1141, 2009.

[2] I. D. Delbem, R. Galéry, P. R. G. Brandão, and A. E. C. Peres, "Semi-automated iron ore characterisation based on optical microscope analysis: quartz/resin classification," Minerals Engineering, vol. 82, pp. 2-13, 2015.

[3] M. T. Sweetapple and S. Tassios, "Laser-induced breakdown spectroscopy (LIBS) as a tool for in situ mapping and textural interpretation of lithium in pegmatite minerals," American Mineralogist, vol. 100, no. 10, pp. 2141-2151, 2015.

[4] C. A. Munson, J. L. Gottfried, E. G. Snyder, F. C. De Lucia Jr., B. Gullett, and A. W. Miziolek, "Detection of indoor biological hazards using the man-portable laser induced breakdown spectrometer," Applied Optics, vol. 47, no. 31, pp. G48-G57, 2008.

[5] J. L. Gottfried, F. C. De Lucia Jr., C. A. Munson, and A. W. Miziolek, "Standoff detection of chemical and biological threats using laser-induced breakdown spectroscopy," Applied Spectroscopy, vol. 62, no. 4, pp. 353-363, 2008.

[6] M. Banaee and S. H. Tavassoli, "Discrimination of polymers by laser induced breakdown spectroscopy together with the DFA method," Polymer Testing, vol. 31, no. 6, pp. 759-764, 2012.

[7] S. J. Rehse, J. Diedrich, and S. Palchaudhuri, "Identification and discrimination of Pseudomonas aeruginosa bacteria grown in blood and bile by laser-induced breakdown spectroscopy," Spectrochimica Acta-Part B Atomic Spectroscopy, vol. 62, no. 10, pp. 1169-1176, 2007.

[8] I. Rehan, K. Rehan, S. Sultana, M. O. ul Haq, M. Z. K. Niazi, and R. Muhammad, "Spatial characterization of red and white skin potatoes using nano-second laser induced breakdown in air," The European Physical Journal Applied Physics, vol. 73, no. 1, Article ID 10701, 2016.

[9] R. W. Campbell, A Conceptual Analysis of Introductory Physic Textbooks, ProQuest, 2008.

[10] Y. Ralchenko, Memorie della Società Astronomica Italiana, vol. 3-8, 2005.

[11] H. H. Koehn, New Mexico Institute of Mining and Technology, 1977.

[12] S. J. Rehse and Q. I. Mohaidat, "The effect of sequential dual-gas testing on laser-induced breakdown spectroscopybased discrimination: application to brass samples and bacterial strains," Spectrochimica Acta Part B: Atomic Spectroscopy, vol. 64, no. 10, pp. 1020-1027, 2009.

[13] R. A. Fisher, "The use of multiple measurements in taxonomic problems," Annals of Eugenics, vol. 7, no. 2, pp. 179-188, 1936.

[14] J. T. Bromberger, L. L. Schott, K. A. Matthews et al., "Association of past and recent major depression and menstrual characteristics in midlife: study of Women's Health Across the Nation," Menopause, vol. 19, no. 9, pp. 959-966, 2012.

[15] C. J. Huberty, Applied Discriminant Analysis, Wiley, New York, NY, USA, 1994.

\section{Competing Interests}

The authors have no competing interests. 

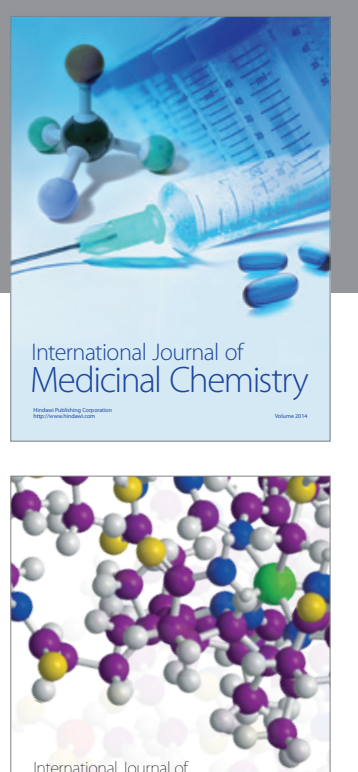

Carbohydrate Chemistry

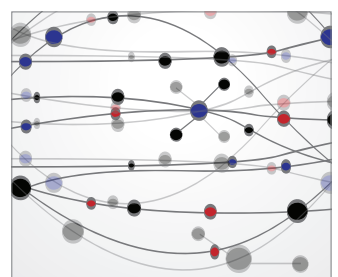

The Scientific World Journal
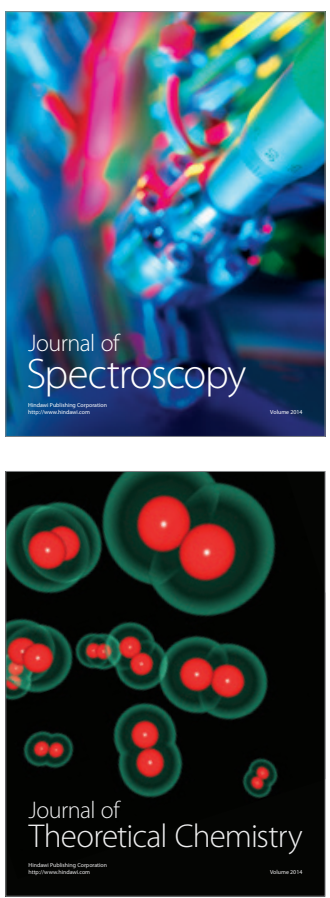
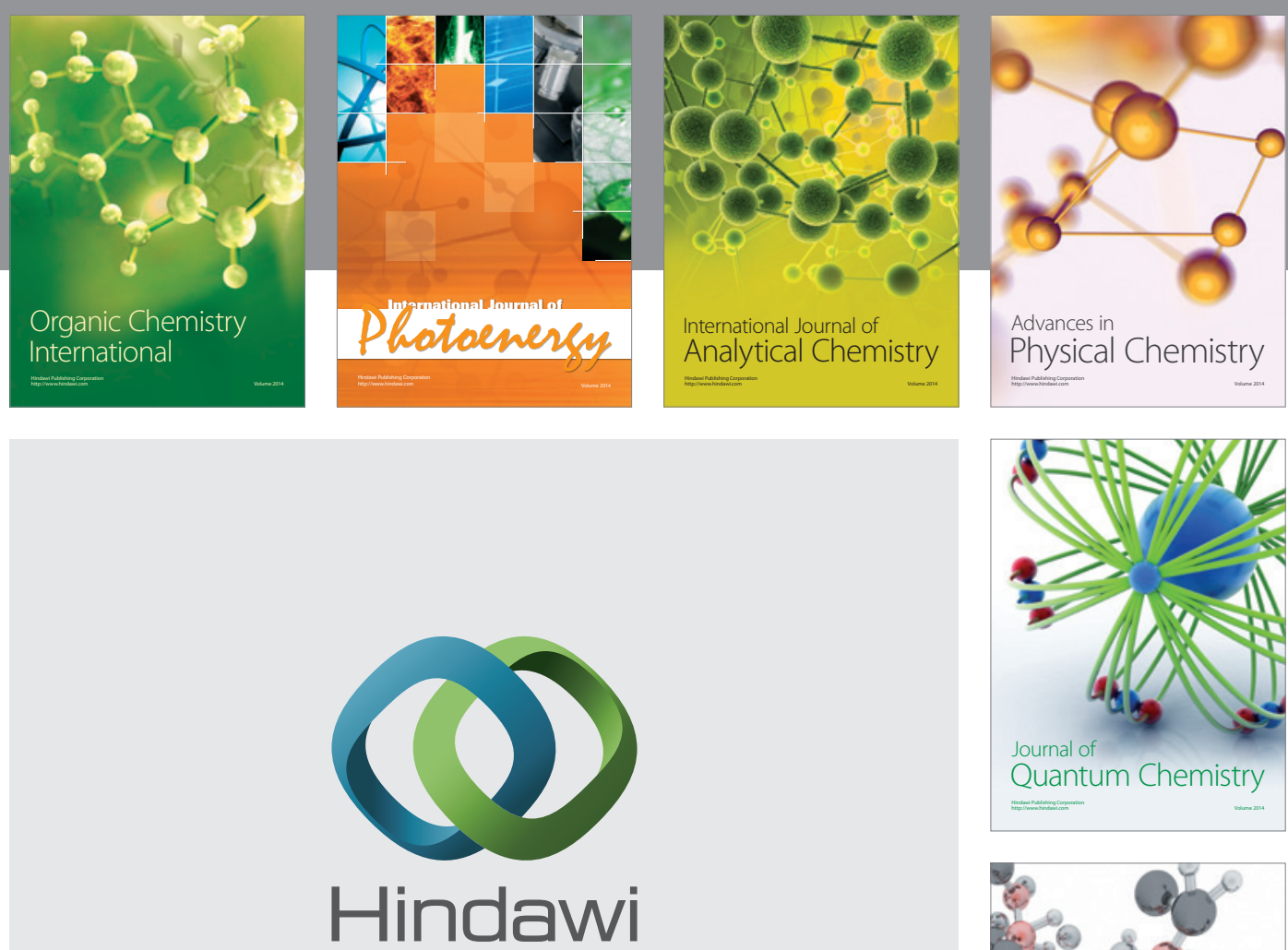

Submit your manuscripts at

https://www.hindawi.com

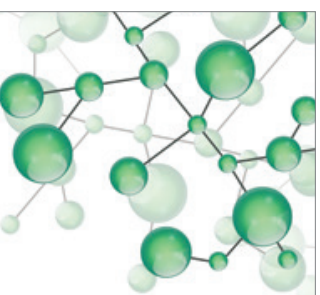

International Journal of

Inorganic Chemistry
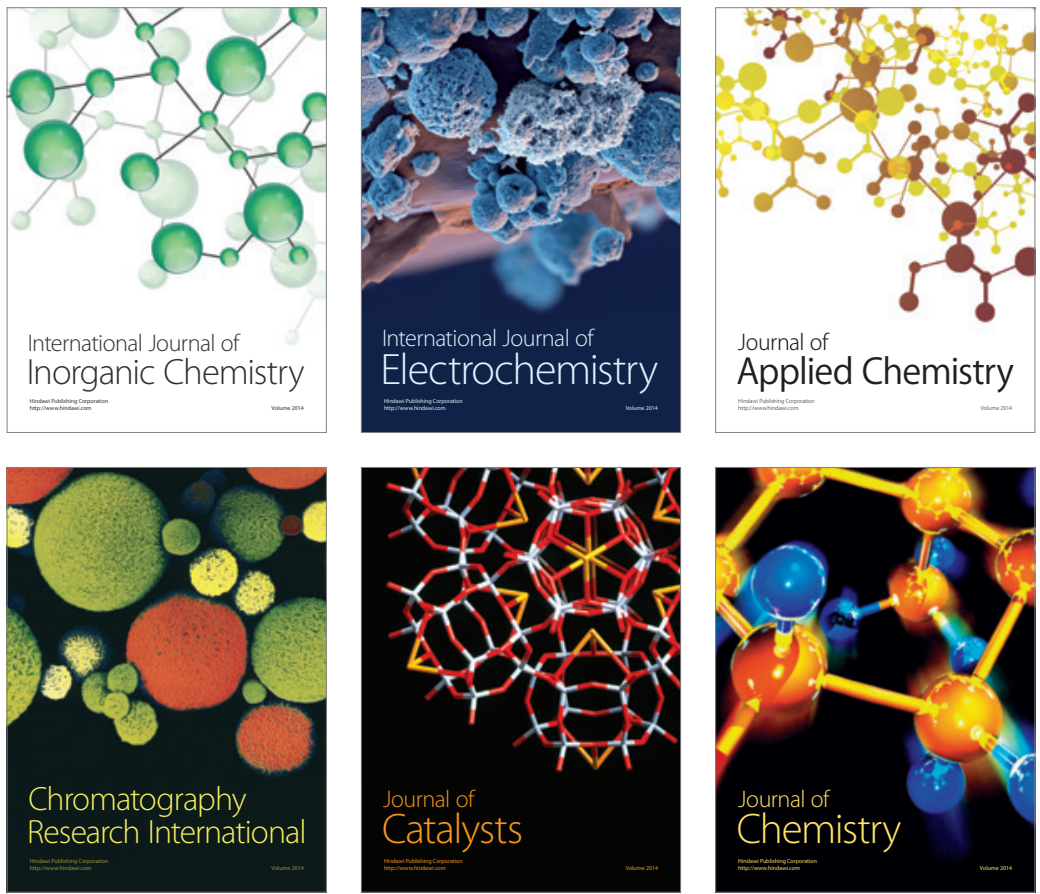

Journal of

Applied Chemistry
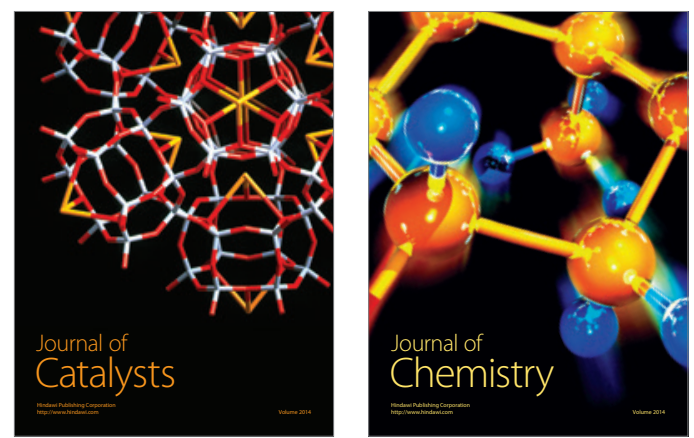
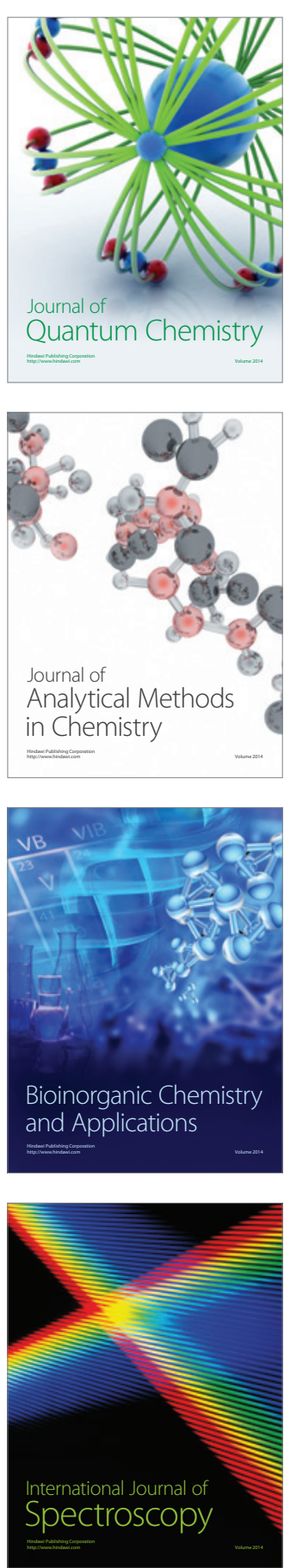